\title{
Aconselhamento em Atividade Física: Uma Proposta de Fluxograma de Intervenção Clínica
}

\section{Physical Activity Counseling: A Proposed Clinical Intervention Flowchart}

Sara Gamboa MADEIRA ${ }^{1,2}$, Patrícia Alexandra SOUSA ${ }^{3}$, Gonçalo ENVIA ${ }^{4}$, Sérgio MARQUES ${ }^{5}$, Tiago Martins MOREIRA $\square^{6}$

Acta Med Port 2018 Jun;31(6):295-298 - https://doi.org/10.20344/amp.9920

Palavras-chave: Exercício; Registos Electrónicos de Saúde; Sinais Vitais

Keywords: Electronic Health Records; Exercise; Vital Signs

\section{INTRODUÇÃO}

Portugal é um dos países da União Europeia com maior taxa de inatividade física, sendo que mais de $60 \%$ da população nunca pratica desporto ou atividade física (AF) de outra natureza. ${ }^{1}$ Segundo a Organização Mundial de Saúde, esta prevalência resulta de atividades laborais e de lazer mais sedentárias, sendo a inatividade física identificada como o quarto fator de risco para a mortalidade global, ${ }^{2}$ à frente da obesidade e da dislipidemia. Neste contexto, a Direção-Geral da Saúde criou um programa prioritário para a Promoção da Atividade Física - PNPAF. ${ }^{3}$

O médico encontra-se numa posição privilegiada para realizar o aconselhamento em AF, tendo como objetivos a melhoria da funcionalidade e qualidade de vida do indivíduo.

Define-se AF como qualquer movimento corporal voluntário que resulte em gasto energético acima do consumo basal em repouso, incluindo as deslocações diárias, atividades domésticas, laborais e de lazer e exercício físico ou desporto. ${ }^{2} \mathrm{Na}$ prática, designa-se por exercício físico a AF realizada de forma estruturada e planeada.

Este artigo pretende sistematizar a abordagem ao aconselhamento em AF no adulto. Optou-se por uma estrutura em sete passos, utilizada em Medicina Geral e Familiar, que acompanha sequencialmente o fluxograma de atuação clínica (Fig. 1).

\section{$1^{\circ}$ Passo: Questionário de atividade física}

O SClínico ${ }^{\circledR}$ dos Cuidados de Saúde Primários tem um questionário de AF que permite avaliar o risco de sedentarismo e o nível de $\mathrm{AF}^{3}$

O risco de sedentarismo é inferido pelo tempo médio diário sentado a ver televisão. Apesar de não estar definido um limiar, a evidência indica que a redução do tempo despendido em comportamentos sedentários tem influência positiva na saúde cardiometabólica e na mortalidade geral. ${ }^{4}$
Este desiderato poderá ser atingido através da promoção de AF não estruturada (ex.: usar escadas, aguardar em pé, estacionar longe e caminhar, pausas ativas).

O nível de AF é avaliado através do conceito 'Exercício como Sinal Vital', que se baseia em duas questões - quantos dias por semana realiza exercício físico pelo menos moderado e quanto tempo dura cada sessão de exercício físico. ${ }^{5} \mathrm{O}$ produto dos resultados permite classificar o indivíduo em fisicamente ativo (realiza pelo menos 150 minutos de exercício físico por semana) ou insuficientemente ativo. Estes últimos deverão ser alvo de aconselhamento.

De notar que mesmo um indivíduo que cumpra as recomendações de exercício físico semanal deve ser alvo de intervenção no âmbito da promoção de AF não estruturada caso passe muitas horas por dia sentado.

\section{$2^{\circ}$ Passo: Disponibilidade para a mudança comporta- mental}

Para definirmos o tipo de estratégia e aconselhamento a realizar, torna-se necessária a categorização do indivíduo segundo o padrão de prática de AF atual e o estádio de mudança comportamental. Para aferir a disponibilidade para a mudança, optou-se por utilizar os estádios de mudança comportamental do Modelo Transteórico de Proschaska e DiClemente (Pré-contemplação, Contemplação, Preparação, Ação, Manutenção) ${ }^{6}$ que devem ser explorados à luz da Entrevista Motivacional.

\section{$3^{\circ}$ Passo: Avaliação médica}

A avaliação médica tem como principais objetivos a identificação de indivíduos com contraindicações médicas absolutas e relativas que necessitem de controlo ou resolução e de indivíduos com patologia que justifique integração em programas de exercício sob supervisão. ${ }^{7}$ Os componentes da avaliação são a anamnese, o exame físico e

\footnotetext{
1. Instituto de Saúde Ambiental. Faculdade de Medicina. Universidade de Lisboa. Lisboa. Portugal.

2. Unidade de Saúde Familiar Mactamã. Agrupamento dos Centros de Saúde de Sintra. Queluz. Portugal.

3. Unidade de Saúde Familiar Cova da Piedade. Agrupamento dos Centros de Saúde de Almada-Seixal. Almada. Portugal.

4. Unidade de Saúde Familiar Lapiás. Agrupamento dos Centros de Saúde de Sintra. Pêro Pinheiro. Portugal.

5. Unidade de Saúde Familiar Servir Saúde. Agrupamento dos Centros de Saúde de Almada-Seixal. Corroios. Portugal.

6. Unidade de Saúde Familiar Pinhal de Frades. Agrupamento dos Centros de Saúde de Almada-Seixal. Seixal. Portugal.

$\square$ Autor correspondente: Tiago Martins Moreira. tiago87moreira@hotmail.com

Recebido: 11 de novembro de 2017 - Aceite: 16 de abril de 2018 | Copyright @ Ordem dos Médicos 2018
} 

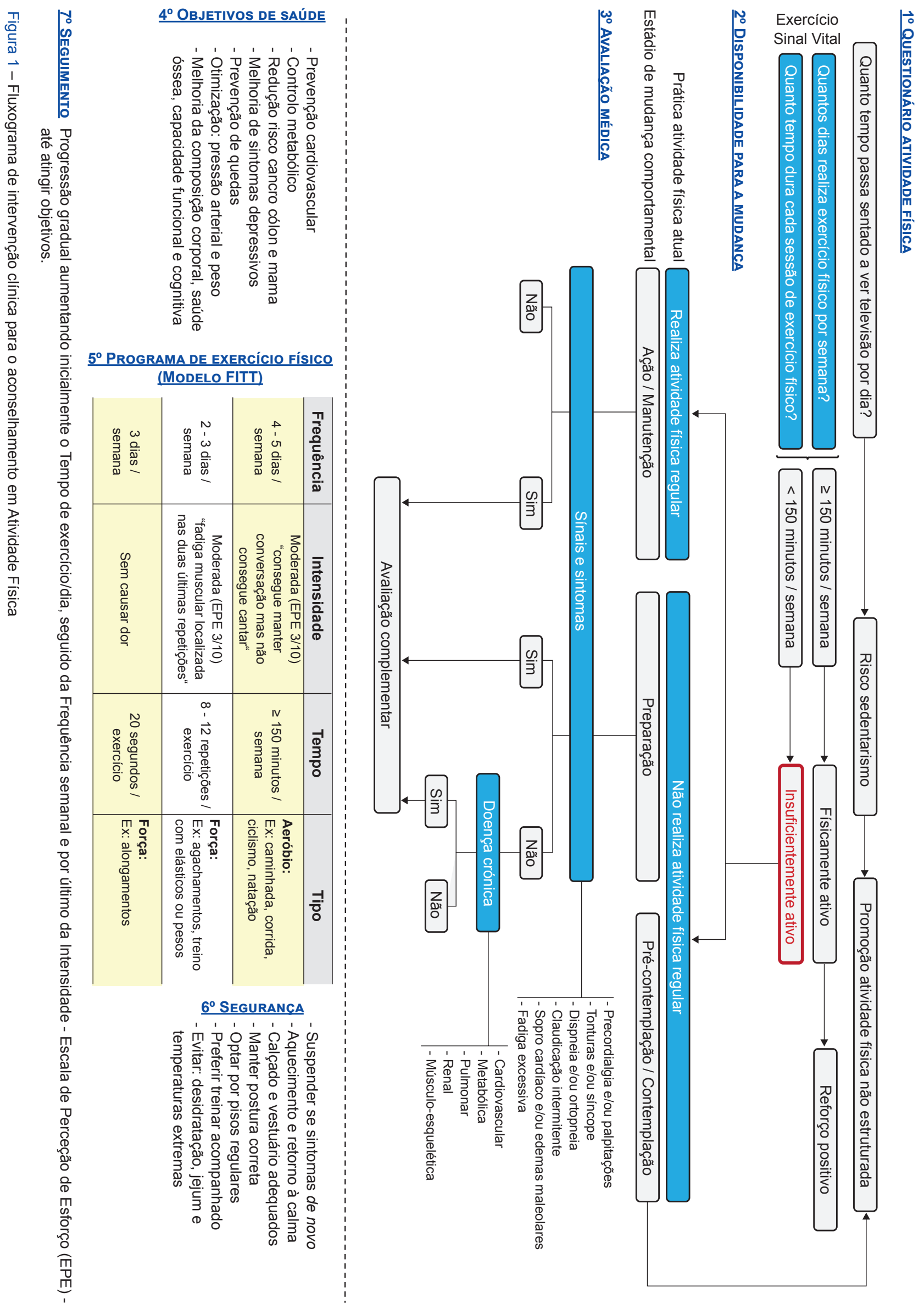
avaliação complementar. Esta pode incluir o pedido de exames complementares de diagnóstico, nomeadamente eletrocardiograma (indivíduos até 35 anos) e prova de esforço (após os 35 anos), ou a avaliação por outra especialidade. No fluxograma estão identificados os sinais, sintomas e patologias mais relevantes a considerar.

\section{$4^{\circ}$ Passo: Objetivos de saúde}

A definição de metas mensuráveis, realistas e individualizadas é fundamental para se adequar o aconselhamento às preferências do indivíduo, aos recursos da comunidade e à presença de condições patológicas prévias, bem como para se avaliar a intervenção efetuada.

Ressalva-se que os benefícios de saúde ${ }^{9}$ explicitados no fluxograma apenas têm evidência em indivíduos que cumpram critérios para fisicamente ativos.

\section{$5^{\circ}$ Passo: Programa de exercício físico (modelo FITT)}

O modelo FITT é um acrónimo dos componentes a incluir na definição de um programa de exercício: 'Frequência', 'Intensidade', 'Tempo' e 'Tipo'. 7,10

Os principais 'Tipos' de exercício são: aeróbio, que melhora a condição cardiorrespiratória; força, que resulta no fortalecimento muscular e deverá englobar os grandes grupos musculares (ex:: músculos dorsais, abdominais, quadricípites); e flexibilidade, que melhora a amplitude dos movimentos articulares.

Quanto à 'Frequência', o exercício aeróbio deve ser realizado na maioria dos dias e distribuído ao longo da semana. Os exercícios de força não devem ser realizados em dias consecutivos mas podem ser acumulados com os outros tipos.

A 'Intensidade' pode ser considerada o aspeto mais relevante de um programa de exercício, uma vez que existe um efeito dose-dependente entre um determinado limiar de intensidade e os seus benefícios. Esta componente deve ser determinada com base numa escala de perceção de esforço (EPE) em alternativa à frequência cardíaca, uma vez que esta pode ser influenciada por muitas patologias crónicas e fármacos. Optou-se pela EPE de Borg modificada (0 - 10), estando definido o exercício de intensidade moderada na pontuação 3 da escala, sendo as pontuações abaixo consideradas intensidade ligeira e acima vigorosa. Para o exercício aeróbio, uma forma simples de aferição de intensidade moderada é a capacidade de manter a conversação mas não conseguir cantar durante o exercício (talk test).

\section{REFERÊNCIAS}

1. TNS Opinion \& Social. Special Eurobarometer 472: sport and physical activity: report. Special Eurobarometer 472. 2018. [consultado 2018 mar 30]. Disponível em: http://ec.europa.eu/commfrontoffice/publicopinion/ index.cfm/survey/getsurveydetail/instruments/special/surveyky/21640.

2. World Health Organization. Global recommendations on physical activity for health. Genève:WHO. 2010. [consultado 2018 mar 30]. Disponível em: http://www.who.int/dietphysicalactivity/publications/9789241599979/ en/.

3. Gabinete do Secretário de Estado Adjunto e da Saúde. Despacho n. ${ }^{\circ}$ 8932/2017. Diário da República n. ${ }^{\circ}$ 195/2017, 2. ${ }^{a}$ série de 10 de outubro
Relativamente à componente 'Tempo', verifica-se que o cumprimento das recomendações de pelo menos 150 minutos semanais de exercício aeróbio deve ser realizado através da acumulação de várias sessões. Estas deverão ter uma duração mínima de 10 minutos consecutivos, o que pode ser uma alternativa para quem tem limitações funcionais ou de disponibilidade. No treino de força, o tempo pode definir-se em número de repetições por exercício.

\section{$6^{\circ}$ Passo: Segurança}

O aconselhamento em AF deve conter informações sobre a importância da supervisão e as potenciais complicações associadas ao exercício. Assim, cada indivíduo deverá conhecer um conjunto de recomendações, sinais de alarme e particularidades da modalidade que tornem a prática segura.

O aquecimento e o retorno à calma devem ser sempre realizados por um período de 5 a 10 minutos em intensidade ligeira. ${ }^{7} \mathrm{O}$ aquecimento faz a transição do estado de repouso para a atividade (ex.: caminhada rápida). O término da AF não deve ser abrupto, incluindo um retorno à calma ativo (ex.: corrida lenta), útil para a recuperação e prevenção de efeitos adversos.

Nos indivíduos descondicionados, pode ser adequado iniciar um programa de exercício com intensidade ligeira mantendo o objetivo de atingir a capacidade para realizar exercícios de intensidade moderada. ${ }^{10}$

\section{$7^{\circ}$ Passo: Seguimento}

Numa consulta de seguimento o aconselhamento deverá ser revisto passo por passo a fim de identificar inconformidades entre o proposto e o alcançado. Estas consultas são importantes por constituírem momentos ótimos para reforço positivo, trabalhar divergências e dificuldades, bem como para discussão multidisciplinar (com o profissional de exercício) sobre formas de progressão dos componentes do modelo FITT.

\section{CONCLUSÃO}

Com este artigo pretende-se sensibilizar os médicos para a importância da prática de $\mathrm{AF}$, tanto nos indivíduos saudáveis como nos portadores de doença crónica e em todas as fases do ciclo de vida. O fluxograma apresentado tem como propósito simplificar o aconselhamento em AF por forma a torná-lo uma prática generalizada nas várias especialidades médicas.

de 2017; 2017. p. 22878-9. [consultado 2017 nov 1]. Disponível em: https://dre.pt/application/conteudo/108275649.

4. Young DR, Hivert MF, Alhassan S, Camhi SM, Ferguson JF, Katzmarzyk PT, et al. Sedentary behavior and cardiovascular morbidity and mortality: a science advisory from the American Heart Association. Circulation. 2016;134:19

5. Sallis R. The physical activity as vital sign and electronic records. Aspetar Sport Med J. 2009;118-23.

6. Prochaska JO, DiClemente CC, Norcross JC. In search of how people change. Am Psychol. 1992;27:1102-14. 
7. Riebe D, Ehrman J, Liguori G, Magal M. ACSM's guidelines for exercise testing and prescription. $10^{\text {th }}$ ed. Philadelphia: Wolters Kluwer; 2018.

8. Maron BJ, Friedman RA, Kligfield P, Levine BD, Viskin S, Chaitman BR, et al. Assessment of the 12-Lead ECG as a screening test for detection of cardiovascular disease in healthy general populations of young people (12-25 years of age). Circulation. 2014;130:1303-34.

9. Lee IM, Shiroma EJ, Lobelo F, Puska P, Blair SN, Katzmarzyk PT, et al. Effect of physical inactivity on major non-communicable diseases worldwide: An analysis of burden of disease and life expectancy. Lancet. 2012;380:219-29.

10. Moore G, Durstine JL, Patricia P. Exercise management for persons with chronic diseases and disabilities. $4^{\text {th }}$ ed. Champaign: ACSM Human Kinetics; 2016. 